\title{
A comparison of different methods for assessing leaf area index in four canopy types
}

\author{
Cristina Ariza-Carricondo ${ }^{1, *}$, Francesca Di Mauro ${ }^{2}$, Maarten Op de Beeck ${ }^{1}$, \\ Marilyn Roland ${ }^{1}$, Bert Gielen ${ }^{1}$, Domenico Vitale ${ }^{2}$, Reinhart Ceulemans ${ }^{1,3}$, Dario Papale ${ }^{2}$ \\ ${ }^{1}$ University of Antwerp, Department of Biology, Research Center of Excellence on Plants and Ecosystems, Universiteitsplein 1, \\ B-2610 Wilrijk, Belgium \\ ${ }^{2}$ University of Tuscia, Department for Innovation in Biological, Agro-food and Forest systems (DIBAF), Via C. de Lellis, \\ 01100 Viterbo, Italy \\ ${ }^{3}$ CzechGlobe, SustES, Belidla 4a, CZ-603 00 Brno, Czech Republic
}

\begin{abstract}
The agreement of Leaf Area Index (LAI) assessments from three indirect methods, i.e. the LAI-2200 Plant Canopy Analyzer, the SS1 SunScan Canopy Analysis System and Digital Hemispherical Photography (DHP) was evaluated for four canopy types, i.e. a short rotation coppice plantation (SRC) with poplar, a Scots pine stand, a Pedunculate oak stand and a maize field. In the SRC and in the maize field, the indirect measurements were compared with direct measurements (litter fall and harvesting). In the low LAI range (0 to 2) the discrepancies of the SS1 were partly explained by the inability to properly account for clumping and the uncertainty of the ellipsoidal leaf angle distribution parameter. The higher values for SS1 in the medium (2 to 6 ) to high (6 to 8 ) ranges might be explained by gap fraction saturation for LAI-2200 and DHP above certain values. Wood area index -understood as the woody lightblocking elements from the canopy with respect to diameter growth-accounted for overestimation by all indirect methods when compared to direct methods in the SRC. The inter-comparison of the three indirect methods in the four canopy types showed a general agreement for all methods in the medium LAI range (2 to 6). LAI-2200 and DHP revealed the best agreement among the indirect methods along the entire range of LAI (0 to 8$)$ in all canopy types. SS1 showed some discrepancies with the LAI-2200 and DHP at low (0 to 2) and high ranges of LAI (6 to 8).
\end{abstract}

Key words: LAI-2200; SunScan; DHP; indirect methods; direct methods; Wood Area Index

Editor: Bohdan Konôpka

\section{Introduction}

Canopy leaf area is a crucial driver of light interception, and thus of photosynthetic carbon uptake and biomass production (GCOS 2011). Leaf area index (LAI), the metric of canopy leaf area, is commonly defined as half of the total leaf area per unit ground surface area (Chen \& Black 1992). LAI is closely related to vegetation-atmosphere interactions as well as to gas exchange processes as photosynthesis and evapotranspiration (Duchemin et al. 2006). Being a key variable in ecological, hydrological and biogeochemical models and a reliable indicator of crop productivity, LAI is used to facilitate the understanding of dynamic vegetation changes and of the impact of climate change on ecosystems (Lin et al. 2016).

Unfortunately, the direct quantification of LAI requires a lot of manual labour as it implies the physical measurement of the area of leaves obtained from the destructive sampling of (parts of the) vegetation or by collecting leaf litter. The destructive sampling of evergreen, especially coniferous, species needs a very premeditated protocol. Morphological properties (e.g. specific leaf area) of needles differ along the vertical crown profile, among needles of different ages as well as among trees of different sizes due to competition pressure (Konôpka \& Pajtík 2014). For tree genera that produce leaves at different time intervals during the growing season, as for example poplars, litter trap data represent an overestimation of the maximum LAI (Jonckheere et al. 2004). Destructive harvesting introduces a permanent disturbance to the canopy and is nearly impossible in forest canopies. It is furthermore not always possible to collect data along the entire season as the collection of leaf litter can only be used to estimate the seasonal LAI maximum and the pattern of LAI during leaf fall. It is therefore dif- 
ficult to assess the spatio-temporal dynamics of LAI using direct measurements.

To overcome these limitations a number of indirect methods have been developed, as described in some comprehensive reviews (Breda 2003; Jonckheere et al. 2004; Zheng \& Moskal 2009). By indirect methods LAI is inferred from observations of a more easily measurable variable. Although in principle less accurate on a sample basis than direct measurements, indirect methods are frequently used since they are faster, allowing for a larger sample size and a higher spatial representativeness. Indirect methods are either based on light interception measurements, hemispherical photography, allometry or remote sensing. The latter approach is the most efficient method for large-scale LAI estimates, but it requires validation with ground truth data. The two first mentioned approaches infer LAI from measurements of the transmission of radiation through the canopy, making use of the radiative transfer theory (for details see Breda (2003) and references therein). They are here referred to as indirect optical methods. Besides hemispherical photography, alternative restricted-angle methods include $57^{\circ}$ photography and zenith cover photography (Macfarlane et al. 2007; Alivernini et al. 2018). Some other recent methods include Terrestrial Laser Scanning (TLS) and Unmanned Aerial Vehicle (UAV) to estimate LAI in forestry (Chianucci et al. 2016; Woodgate et al. 2015).

Indirect optical methods are widely used to estimate LAI in a range of canopy types (forests, croplands, grasslands, etc.), but they have some limitations. First of all, they do not really measure LAI but rather plant area index (PAI), since radiation is intercepted by all light-blocking plant parts. In other words, these methods do not distinguish photosynthetically active (green) leaf tissue from non-green or woody plant parts as stems, branches or flowers. They hence tend to overestimate the true LAI in canopies where non-green plant parts are present (Weiss et al. 2004; Zheng \& Moskal 2009). A second important concern is the clumping, i.e. the spatial aggregation of plant elements in the canopy. As leaves are not randomly distributed within a forest canopy, the clumping index describes the extent to which LAI retrieved from a random model of leaf placement differs from true LAI (Zhao et al. 2012). Although indirect methods are very useful and widely applicable, each one has its own inherent bias and errors, and is therefore more suited for particular conditions than other methods. To provide more reliable and more accurate LAI measurements with indirect methods, a range of techniques and correction procedures have been developed. These have been compared in a number of studies, on crops (Facchi et al. 2010, Fang et al. 2018), on rice (Fang et al. 2014), on forest stands (Macfarlane et al. 2007; Ryu et al. 2010b; Chianucci \& Cutini 2013), on pine (Mason et al. 2012) and for different rice varieties (Sone et al. 2009). For a mix of canopies different - but only indirect - methods were compared (Woodgate et al. 2015). The current research of this manuscript differs in that these afore-mentioned studies focused either on the comparison of different instruments tested for the same canopy, or on the comparison of different canopies with the same instrument. The present study combines these factors, i.e. four canopies were studied, three different indirect methods were compared (LAI-2200 Plant Canopy Analyzer, SunScan Plant Canopy Analysis System and Digital Hemispherical Photography) and two direct methods were used to validate the results (litter fall collection and harvesting). This reflects the aim of the present study, i.e. assessing the agreement of the above-mentioned indirect methods to better understand their bias and to quantify the error terms for different canopy types.

\section{Materials and methods}

\subsection{Description of sites and plant material}

Four different canopy types - all located in Belgium were selected for this study, i.e. a short rotation poplar coppice (SRC) plantation, a mature Scots pine stand, a mature Pedunculate oak stand, and a maize field. Abrief summary of the site characteristics including location, vegetation type, year of most recent thinning or harvest, and planting density is presented in Table 1.

The short rotation coppice (SRC) plantation is located in Lochristi, province East of Flanders. In April 2010 an area of 14.5 ha was planted at a density of 8000 trees ha ${ }^{-1}$ with dormant hardwood cuttings of 12 selected genotypes of Populus deltoides, P. maximowiczii, $P$. nigra, $P$. trichocarpa and interspecific hybrids. The genotypes were arranged in large $(0.16-0.61 \mathrm{ha})$ mono-genotypic blocks of eight double rows wide, with alternating distances of $0.75 \mathrm{~m}$ and $1.50 \mathrm{~m}$ between the rows and $1.1 \mathrm{~m}$ between the individual trees within the row. The plantation was harvested for the first time in February 2012. From then on, trees continued to grow as a coppice culture with multiple shoots per stool in the following biennial rotation. The second harvest took place in February 2014. More detailed information about the site history, soil type, management procedures and productivity has been previously published (Broeckx et

Table 1. Main characteristics of the four canopy types and study sites selected for this study.

\begin{tabular}{|c|c|c|c|c|}
\hline & Short Rotation Coppice & Scots pine & Pedunculate oak & Maize field \\
\hline Location & Lochristi, East-Flanders & Brasschaat, Antwerp & Brasschaat, Antwerp & Wilrijk, Antwerp \\
\hline Coordinates (deg. N, deg. E) & $51.1122,3.8505$ & $51.3092,4.5205$ & $51.3092,4.5205$ & $51.1476,4.4161$ \\
\hline Species description & $\begin{array}{c}\text { Populus deltoides, P. maximowiczii, } \\
\text { P. nigra, P. trichocarpa }\end{array}$ & Pinus sylvestris & Quercus robur & Zea mays, var GL Fantastic \\
\hline Density (trees ha ${ }^{-1}$; seeds ha ${ }^{-1}$ ) & 8000 & 360 & 310 & 94000 \\
\hline Thinning or Harvest & 2014 & 2015 & No recent data & 2015 \\
\hline
\end{tabular}


al. 2012; Verlinden et al. 2015; see also http://uahost. uantwerpen.be/popfull/).

The Scots pine (Pinus sylvestris L.) stand is located in the urban mixed forest 'De Inslag' (150 ha) in Brasschaat, province of Antwerp. The selected stand is about 1.7 ha with an overstory of Scots pine and an understory of mosses, grasses (Molinia caerulea [L.] Moench), Betula pendula Roth and young Scots pine seedlings (Curiel Yuste et al. 2005). It was originally planted in 1929 and regularly thinned and managed since then. In 2011 the stock density was 360 trees ha ${ }^{-1}$ while diameter at breast height and tree height were on average $33 \mathrm{~cm}$ and $21.4 \mathrm{~m}$, respectively (Gielen et al. 2013). The stand canopy is sparse and according to assessments in 2007 the LAI was $1.31 \mathrm{~m}^{2} \mathrm{~m}^{-2}$ with only two needle age classes present (Op de Beeck et al. 2010). In August 2015 the stand was most recently thinned, reducing the number of trees with about one third (Gebauer et al. 2015). Both the SRC plantation and the Scots pine stand host eddy covariance monitoring stations that are part of the European ICOS infrastructure network (www.icos-etc.eu).

The Pedunculate oak (Quercus robur L.) stand is also located in the forest 'De Inslag', close to the Scots pine stand. The oaks were planted in 1936 with a current density of 310 trees ha ${ }^{-1}$. The most recent assessments (2005) reported that canopy height was on average $26 \mathrm{~m}$ (Curiel Yuste et al. 2005). No thinnings were made in this stand.

The maize (Zea mays L.) field was located in Wilrijk, Antwerp. It was cultivated by a local dairy farmer for silage production. The maize crop (variety GL Fantastic) was sown on 5 May 2015 after application of manure $\left(60 \mathrm{~m}^{3} \mathrm{ha}^{-1}\right)$ and ploughing the field to $25 \mathrm{~cm}$ depth. The date of seedling emergence was 20 May 2015. The planting design comprised an inter-row distance of $70 \mathrm{~cm}$ and an average distance between plants in the row of $14 \mathrm{~cm}$. The sowing density was about 94,000 seeds ha ${ }^{-1}$ with a SW-NE row direction.

\subsection{Indirect optical methods}

Three widely applied instruments for indirect LAI measurements were used in this study, i.e. the LAI-2200 Plant Canopy Analyzer (LI-COR ${ }^{\circledR}$, Lincoln, NE, USA), the SS1 SunScan ceptometer (Delta-T Devices Ltd, Cambridge, UK) and Digital Hemispherical Photography (DHP). The theory of leaf area index calculation has been explained in detail in the Appendix text 1 . Theory of leaf area index calculation.

\subsubsection{LAI-2200 Plant Canopy Analyzer}

The LAI-2200 Plant Canopy Analyzer (LI-COR ${ }^{\circledR}$, Lincoln, NE, USA), further referred to as LAI-2200, measures the canopy transmittance of diffuse light at five zenith angles, which is obtained from simultaneous measurements of diffuse light above and below the canopy with a fish-eye PAR sensor divided in five concentric rings. LAI is estimated by inverting the measured canopy transmittance (Eq. 1 in Appendix text 1) and taking into account the Apparent Clumping Factor (ACF) under the usual assumption that leaves are randomly (Poisson) distributed in the canopy (cfr. user manual of the LAI-2200 Plant Canopy Analyzer).

In this study the below-canopy measurements were manually taken with one LAI-2200 sensor, while abovecanopy measurements were collected with another LAI-2200 sensor installed in a clearing close to the site. This sensor was set to automatically log incoming light readings at a $30 \mathrm{~s}$ interval during below-canopy measurements, which were afterwards matched to the closest readings in time. To avoid any influence from the operator the standard five ring configuration was used with a $45^{\circ}$ view cap on both above- and below-canopy sensors. All LAI-2200 measurements were processed with the FV2200 software (v 1.0.0).

\subsubsection{SS1 SunScan Plant Canopy Analysis System}

The SS1 SunScan Plant Canopy Analysis System (DeltaT Devices, Cambridge, UK), further referred to as SS1, is a ceptometer that uses measurements of radiation transmittance through the canopy to provide LAI estimates by applying the Beer-Lambert extinction law (Eq. 3 in Appendix text 1) and taking into account other parameters as absorption and transmission of diffuse light, and zenith angles ( $c f r$. user manual of the SunScan Canopy Analysis System version 3.3). This method requires simultaneous measurements of both incident and transmitted photosynthetically active radiation (PAR), a requirement that was met in different ways depending on the canopy type. For the SRC, the Scots pine and the Pedunculate oak stands, two instruments were used that independently collected above- and below-canopy measurements, similar to the methodology explained above for the LAI-2200. In the maize crop only one instrument was used. This instrument was radio connected to an external PAR sensor measuring incoming PAR, allowing for simultaneous above- and below-canopy PAR readings.

The SS1 SunScan ceptometer uses a specific term to define the Ellipsoidal Leaf Angle Parameter (ELADP). This parameter is modified depending on the canopy and characterizes the horizontal or vertical orientation of the leaves (cfr. user manual of the SunScan Canopy Analysis System version 3.3). Because site-specific ELADPvalues were not available, ELADP was set to 1 for the SRC and the Scots pine and Pedunculate oak stands, and set to 1.37 for the maize crop. The SS1 does not account for clumping. 


\subsubsection{Digital Hemispherical Photography (DHP)}

With this method, which is also known as fish-eye photography and further referred to as DHP, LAI is derived with the inverted Poisson model (Eq. 2 in Appendix text 1) from the amount and the distribution of vegetation pixels on digital hemispherical pictures of the canopy. In this study, the hemispherical pictures were first transformed into binary black and white pictures using the Ridler-Calvard thresholding algorithm (Ridler \& Calvard 1978). Gap fraction was estimated and LAI was calculated by inversion of the Poisson model as described by Thimonier et al. (2010), and by accounting for clumping using the logarithmic averaging method (Lang \& Xiang 1986). All the above calculations are implemented in the HemiTool software, which is the standard processing protocol for hemispherical imagery as a standard LAI measurement method at all ecosystem stations of the ICOS research infrastructure (www.icos-etc.eu).

All hemispherical pictures were taken with a digital single-lens reflex camera (DSLR; Nikon D7100, Tokyo, Japan) in combination with a hemispherical lens (4.5 mm F 1:2.8 DC HSM Sigma Corporation, New York, USA). Following the ICOS measurement protocol, pictures were made in RAW format, using the auto-focus of the lens, a fixed aperture of 8 , and the lowest shutter speed at which no overexposure occurred. The minimum camerato-foliage distance was $15 \mathrm{~cm}$ from the closest leaf or 10 times the leaf length.

\subsection{Sampling design}

All LAI measurements with the three indirect methods were collected over the span of one full growing season, from spring 2015 to spring 2016. Measurement dates were selected to collect data over the full environmental range of LAI values for each canopy type, including the seasonal minima and maxima. Due to some instrument failure and periods of unfavourable weather conditions, it was unfortunately not possible to measure with all three indirect methods at all scheduled dates. All indirect measurements were done under fully overcast sky conditions, i.e. in the absence of direct sunlight. In the SRC and the maize field, also direct LAI measurements were collected.

\subsubsection{Short rotation coppice poplar plantation}

Measurements in the SRC plantation were carried out in three permanent plots of $3 \mathrm{~m}$ by $4 \mathrm{~m}$ inside each of eight selected blocks, each block representing a different poplar genotype. At each measurement date 12 below-canopy readings were made in each plot with the LAI-2200 and SS1, six parallel and six perpendicular to the rows (Fig. 1a).

Above-canopy readings for both instruments were taken in a nearby clearing. In each of the $3 \times 8$ plots two hemispherical pictures were taken parallel to the rows with the camera positioned as close to the ground as possible (Fig. 1a). Measurements were taken about every two weeks between the time of bud break and the end of leaf fall, as well as one measurement during the leafless period in winter.
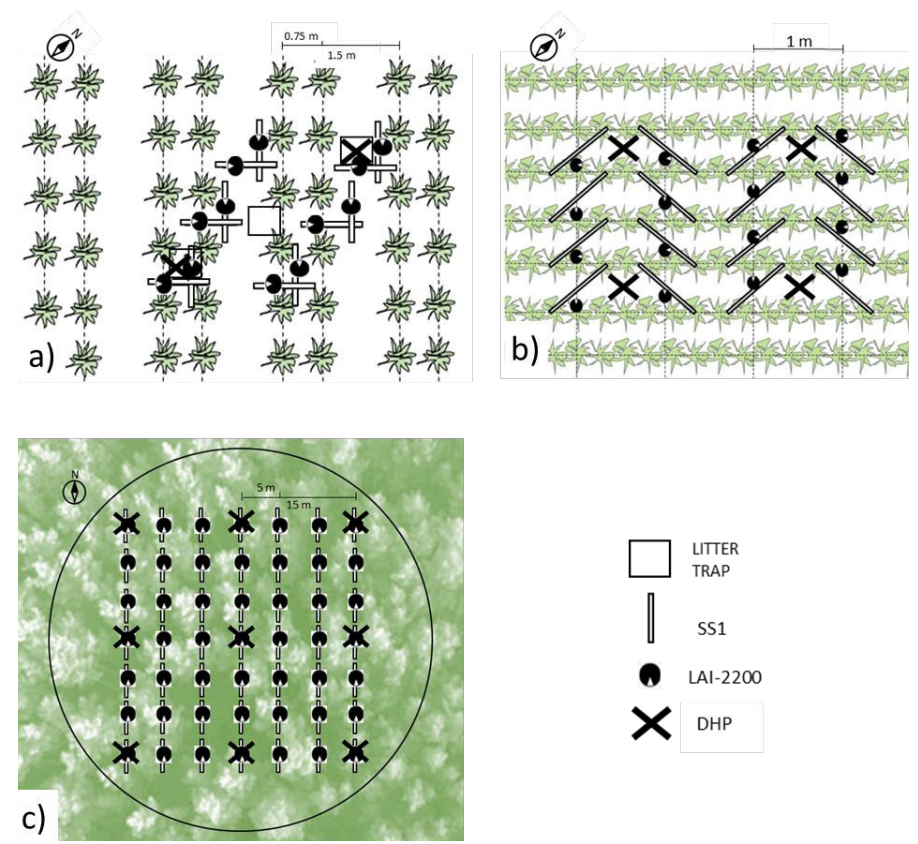

Fig. 1. Description of the sampling design in the different ecosystems. Measurements were performed in a short rotation coppice poplar plantation (a), in a maize field (b) and in a mature Scots pine and a mature Pedunculate oak stand (c). Location of the three indirect measurements (LAI-2200, SS1 and DHP) are reported in all ecosystems and litter trap positions in the short rotation coppice. 
Direct LAI measurements in the SRC plantation were obtained with the gravimetric method (Daughtry 1990) from leaf litter collected at regular intervals from the start to the end of the leaf fall period, i.e. from late August to December 2015. In each plot, leaf litter was collected in three plastic litter traps (surface area of $0.22 \mathrm{~m}^{2}$ ) installed along a diagonal transect (Fig. 1a). Collected leaves were transported to the laboratory, dried in a drying oven at $70^{\circ} \mathrm{C}$ until constant weight and then weighed. Total leaf area (LA) was calculated from dry weight using specific leaf area (SLA), which was determined from a representative sample of nine leaves collected from each plot at the time of maximum LAI $\left(\mathrm{LAI}_{\text {max }}\right)$. SLA was calculated by dividing fresh leaf area of the sample by its dry weight. For each plot LAI was obtained by dividing the total LA by the total ground surface area of the three litter traps inside the plot (Broeckx et al. 2015).

\subsubsection{Scots pine and Pedunculate oak forest stands}

Indirect measurements in the Scots pine and the Pedunculate oak stands were carried out in a $25 \mathrm{~m}$ radius circular plot (Fig. 1c). At each measurement date 49 belowcanopy measurements were made with both LAI-2200 and SS1, one measurement spaced $5 \mathrm{~m}$ apart from the other, and with the sensor always pointing to the South. Simultaneous above-canopy measurements were taken in a nearby clearing. Nine upward-facing hemispherical pictures were taken at $1.3 \mathrm{~m}$ above the ground, the upside of the picture placed to the North, with $15 \mathrm{~m}$ distance between each picture taken. No direct measurements of LAI were collected in these two forest stands.

\subsubsection{Maize field}

Indirect measurements in the maize field were taken in a $3 \mathrm{~m}$ by $3 \mathrm{~m}$ plot almost every two weeks. At each measurement date 16 measurements were taken with the LAI-2200 and SS1 in each plot, as well as four upward looking hemispherical pictures parallel to the row direction (Fig. 1b).

Direct LAI values were obtained from destructive harvesting during the summer period. In each plot 15 plants (five plants in three rows) were removed, and leaves and the stem of the fresh plants were separated. The height of each stem was measured with a ruler and the diameter measured with a calliper at $10 \mathrm{~cm}$ above the ground and at $2 / 3$ of the total stem height. The hemi-surface area of each stem was derived from these measurements assuming a cylindrical stem shape. To calculate LA, total fresh weight of the leaves was first determined, and then the LA of a subsample of approximately $10 \%$ of these leaves was measured with a leaf area meter (LI-3100A, LI-COR ${ }^{\circledast}$, Lincoln, NE, USA). This value was multiplied with the fresh weight ratio of the full sample to the subsample.
The total hemi-surface area of the 15 harvested plants was obtained by summing the LA and the summed hemisurface area of the stems. To obtain the direct LAI estimate, this value was multiplied with the ratio of the total number of plants in the plot to the number of harvested plants and then divided by the ground area of the plot.

\subsection{Statistical analyses}

The results from the three indirect methods and the direct method were pairwise compared for each of the four canopy types. The level of agreement between each pair of methods was assessed by means of robust statistical tools developed for method comparisons, and consisting in: (i) the regression approach of Passing \& Bablok (1983) and (ii) the Tukey mean-difference plot popularised by Bland \& Altman (1986) in analytical chemistry and biostatistics. More details about the statistical methods are to be found in the Appendix text 2.

\section{Results}

This section shows the results of each statistical analysis, i.e. Passing \& Bablok (P-B), Bland and Altman (B-A), Bravais-Pearson correlation coefficient (r) and scatter plots. For reasons of simplicity, the LAI ranges were arbitrarily divided in the following order: low ( 0 to $\left.2 \mathrm{~m}^{2} \mathrm{~m}^{-2}\right)$, medium ( 2 to $\left.6 \mathrm{~m}^{2} \mathrm{~m}^{-2}\right)$ and high $\left(6\right.$ to $\left.8 \mathrm{~m}^{2} \mathrm{~m}^{-2}\right)$ LAI range. The inter-comparison of the indirect methods (LAI-2200, SS1 and DHP) in the four canopies (SRC, Scots pine, Pedunculate oak and maize field) is first broken down (Fig. 2). Afterwards the comparison between the indirect and direct methods (litter fall and harvest) was performed for validation in SRC, and maize field only (Fig. 3).

\subsection{Passing-Bablok regression results between indirect and direct methods}

Focusing on the $95 \%$ CIs of the P-B regression terms for the comparison of indirect methods, the LAI-2200 and SS1 methods were the most comparable in the Pedunculate oak stand and the maize crop, as they provided similar LAI values based on the 95\% CI of intercept and slope coefficients. The LAI-2200 and DHP methods agreed very well in the Scots pine stand and in the SRC. Compared with the direct method, LAI-2200 and DHP readings in the maize field provided unbiased LAI measurements (Table 2). For all other indirect methods there was either a constant bias (when the intercept differed significantly from 0 ), or a proportional bias (when the slope differed significantly from 1 ), or both biases were jointly detected (Fig. 2). These afore-mentioned results need, however, to be interpreted with care because the assumptions underlying the linear regression model were not always met. 

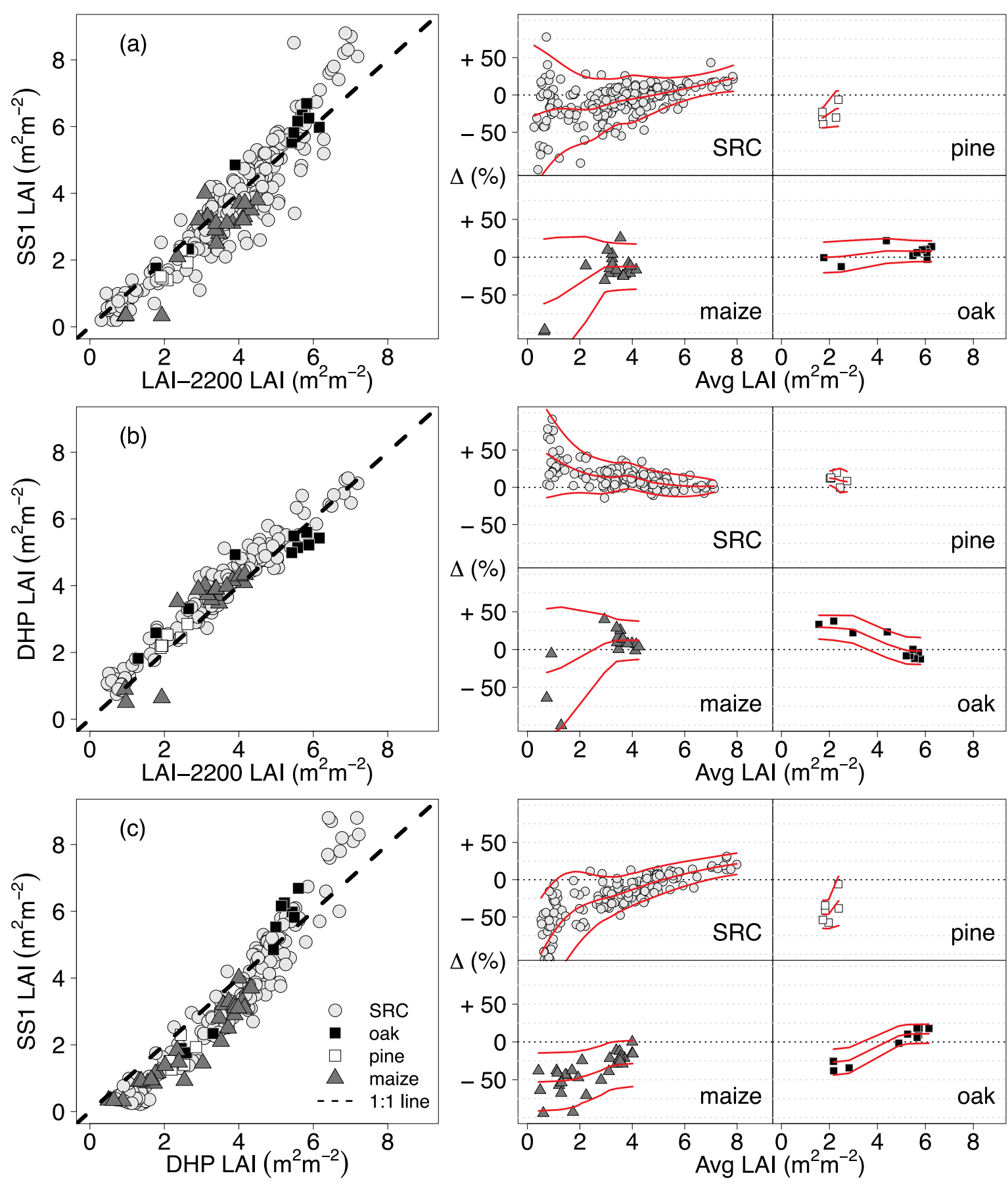

Fig. 2. Inter-comparison of indirect methods for leaf area index (LAI) estimations. Panels on the left $(\mathrm{a}-\mathrm{c})$ show the conventional $\mathrm{x}-\mathrm{y}$ scatter-plots with LAI readings. Panels on the right show the Bland and Altman (B-A) plots separately for each canopy type and for each pair of methods. The horizontal axis in the B-A plots represents the averages of each pair of measurements, while the vertical axis indicates the percentage differences $(\Delta)$ for each pair of measurements, explaining an overestimation or underestimation if the percentage is positive or negative, respectively. Red smoothed lines denote the systematic error (bias) and limits of agreement. SRC: short rotation coppice. 

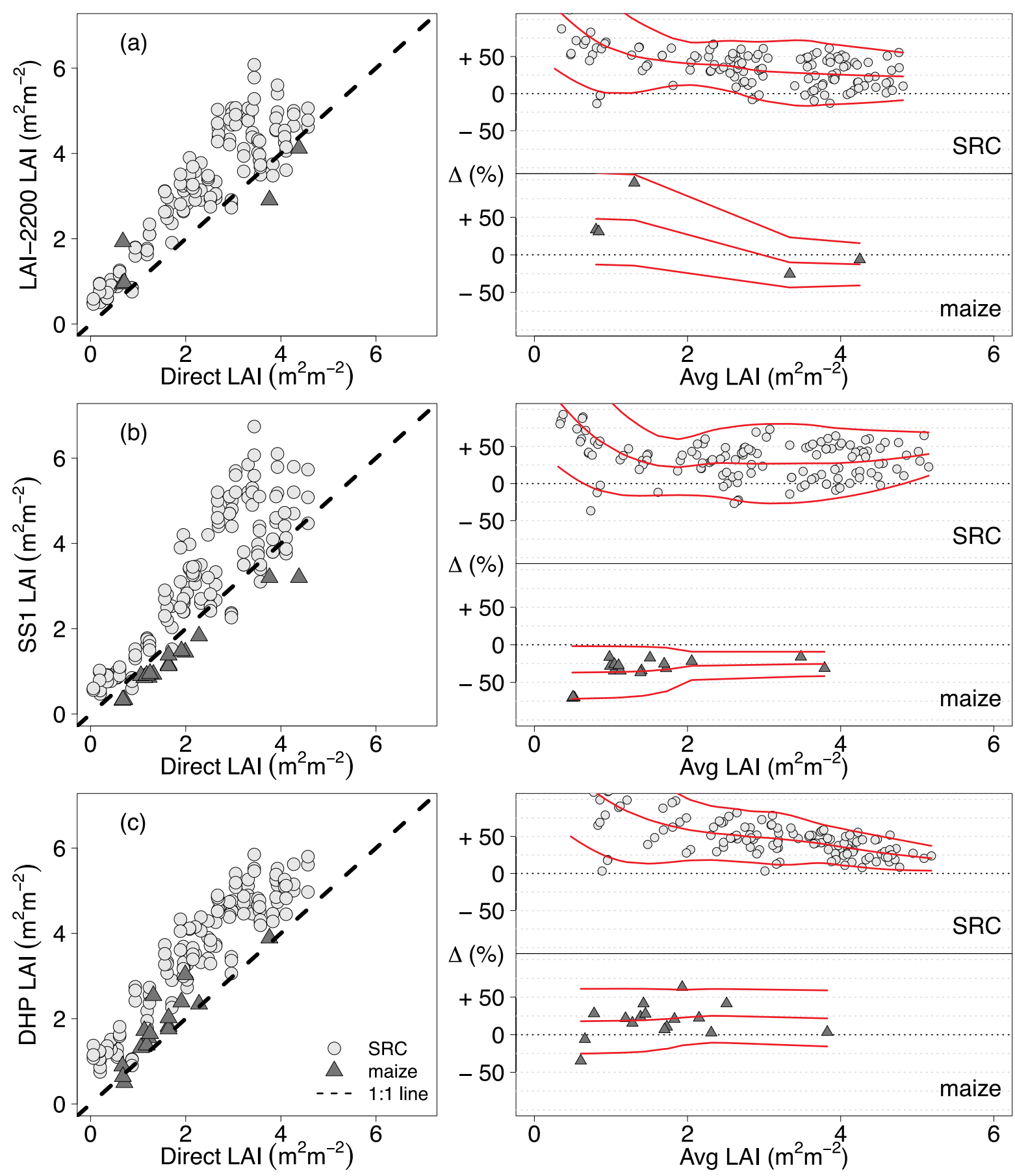

Fig. 3. Comparison of indirect $v s$. direct methods for leaf area index (LAI) estimations. Panels on the left (a-c) show the conventional $x-y$ scatter-plots with LAI readings. Panels on the right show the Bland and Altman (B-A) plots separately for each canopy type and for each pair of methods. The horizontal axis in the B-A plots represents the averages of each pair of measurements, while the vertical axis indicates the percentage differences $(\Delta)$ for each pair of measurements, explaining an overestimation or underestimation if the percentage is positive or negative, respectively. Red smoothed lines denote the systematic error (bias) and limits of agreement. SRC: short rotation coppice. 
Table 2. Results of the statistical analyses used for the inter-comparison between the three indirect methods and the comparison of the indirect with the direct methods (litter fall and harvest) in the four canopies studied (SRC, Scots pine, Pedunculate oak and maize field). The Bias column represents the constant (C) and proportional (P) systematic errors within their associated $95 \%$ confidence interval $(\mathrm{CI})$ after judgement of the 0 and 1 values for the intercept and slope. $\mathrm{N}$ denotes the number of paired observations. Pearson's correlation (r) and intercept and slope coefficients of the simple linear regression $Y=a+b X$ were estimated through the Passing and Bablok (P-B) non-parametric procedure. Est, Low and Upp columns report the estimated, the lower and the upper 95\% (CI), respectively, associated with intercept and slope. SRC: short rotation coppice.

\begin{tabular}{|c|c|c|c|c|c|c|c|c|c|c|}
\hline \multirow{2}{*}{ Xvs Y } & \multirow{2}{*}{ Canopy } & \multirow{2}{*}{ Bias } & \multirow{2}{*}{$\mathrm{N}$} & \multirow{2}{*}{$\mathrm{r}$} & \multicolumn{3}{|c|}{ Intercept } & \multicolumn{3}{|c|}{ Slope } \\
\hline & & & & & Est & Low & Upp & Est & Low & Upp \\
\hline \multirow{4}{*}{ LAI-2200 vs SS1 } & Short Rotation Coppice & $\mathrm{C}+\mathrm{P}$ & 251 & 0.94 & -0.54 & -0.70 & -0.39 & +1.15 & +1.11 & +1.20 \\
\hline & Scots pine & - & 5 & 0.82 & -0.91 & n.a. & n.a. & +1.21 & n.a. & n.a. \\
\hline & Pedunculate oak & - & 10 & 0.97 & -0.40 & -5.10 & +2.02 & +1.16 & +0.71 & +2.01 \\
\hline & Maize field & - & 21 & 0.88 & -0.53 & -1.59 & +0.75 & +0.96 & +0.64 & +1.27 \\
\hline \multirow{4}{*}{ LAI-2200 vs DHP } & Short Rotation Coppice & $\mathrm{C}$ & 143 & 0.98 & +0.49 & +0.35 & +0.63 & +0.96 & +0.93 & +1.00 \\
\hline & Scots pine & - & 6 & 0.88 & +0.31 & -2.50 & +2.32 & +0.97 & +0.00 & +2.41 \\
\hline & Pedunculate oak & $\mathrm{C}+\mathrm{P}$ & 10 & 0.96 & +1.21 & +0.58 & +2.32 & +0.72 & +0.50 & +0.88 \\
\hline & Maize field & - & 17 & 0.91 & +0.54 & -0.64 & +2.21 & +0.93 & +0.48 & +1.28 \\
\hline \multirow{4}{*}{ DHPvs SS1 } & Short Rotation Coppice & $\mathrm{C}+\mathrm{P}$ & 171 & 0.96 & -1.08 & -1.24 & -0.94 & +1.21 & +1.16 & +1.27 \\
\hline & Scots pine & - & 6 & 0.52 & -0.87 & n.a. & n.a. & +1.02 & n.a. & n.a. \\
\hline & Pedunculate oak & $\mathrm{C}+\mathrm{P}$ & 10 & 0.98 & -2.41 & -5.65 & -1.16 & +1.60 & +1.29 & +2.26 \\
\hline & Maize field & $\mathrm{C}$ & 29 & 0.95 & -0.53 & -0.84 & -0.25 & +0.94 & +0.84 & +1.06 \\
\hline LAI-2200 vs Litter fall & Short Rotation Coppice & $\mathrm{C}+\mathrm{P}$ & 134 & 0.90 & +0.47 & +0.29 & +0.61 & +1.18 & +1.08 & +1.31 \\
\hline LAI-2200 vs Harvest & Maize field & - & 5 & 0.93 & +0.40 & n.a. & n.a. & +0.80 & n.a. & n.a. \\
\hline$\overline{\text { DHP vs Litter fall }}$ & Short Rotation Coppice & $\mathrm{C}+\mathrm{P}$ & 133 & 0.93 & +0.98 & +0.84 & +1.11 & +1.12 & +1.04 & +1.20 \\
\hline DHP vs Harvest & Maize field & - & & 0.91 & -0.16 & -0.83 & +0.29 & +1.33 & +0.96 & +1.92 \\
\hline
\end{tabular}

n.a. indicates not available value occurring when the procedure failed to converge because of small sample size.

$r$ as well as the intercept and slope of the simple linear regression model were estimated according to the non-parametric P-B procedure.

\section{2. $\mathrm{X}-\mathrm{Y}$ scatter plot results between indirect and direct methods}

The conventional $x-y$ scatter plots for pairwise comparison of methods (left panels of both Figs. 2 and 3), showed some clear curvature. Among the indirect methods the best agreement was found between the LAI-2200 and DHP, as reflected by the data points close to the 1:1 line (Fig. 2b). The comparison of SS1 with the two other indirect methods yielded a more exponential curve, deviating from the 1:1 line (Fig. 2a and c). The comparison between the direct and the indirect methods showed a general overestimation in SRC and maize field canopies by all indirect methods, especially in the higher LAI range and in the SRC.

\subsection{Bland and-Altman results between indirect and direct methods per canopy type}

The B-A analysis for the inter-comparison of the indirect methods is depicted separately for each canopy type (right panels of Fig. 2). The main results are reported for each canopy type in terms of bias and of Limits of Agreements (LoA). Note that for low LAI values, small absolute differences resulted in high relative variations (in \%), and consequently the LoA diverged.

First, in the SRC, the LAI measurements obtained with DHP and LAI-2200 agreed best, especially in the high LAI range, characterized by a low bias and narrow LoA. In the low-to-medium LAI range, however, DHP produced higher values than LAI-2200; some source of bias as well as diverging LoA were observed. SS1 yielded slightly lower values than LAI-2200 and DHP in the low and medium LAI ranges (up to $4-5 \mathrm{~m}^{2} \mathrm{~m}^{-2}$ ), but higher values in the high LAI range. Secondly, in the Peduncu- late oak stand, a good level of agreement was observed between SS1 and LAI-2200 (low bias and relatively narrow LoA). Measurements with DHP in the low-tomedium LAI ranges were higher than the measurements obtained with SS1 and LAI-2200.

Thirdly, in the Scots pine stand, LAI-2200 and DHP were again in better agreement than compared to SS1, which produced lower values than the other two methods. Due to some instrument failure only few data were available for LAI-2200 in the low LAI range leading to a poor method comparability in the Pedunculate oak and in the Scots pine stands. Finally, in the maize field the three inter-comparisons showed the same tendency, i.e. that LoA diverged with decreasing LAI.

The B-A analysis was also performed for the comparison of the indirect and the direct methods in both the SRC and the maize field (Fig. 3, right panels). In the SRC all three indirect methods overestimated the direct method. The most biased indirect method was the SS1 method, showing a positive bias of $50 \%$ at low LAI values, which decreased to about $25 \%$ in the medium-to-high LAI range. On the contrary, LAI-2200 and DHP methods showed a decreasing bias when LAI increased. The bias in the indirect measurements corresponded to an average overestimation of the direct measurements with 0.5 to $2 \mathrm{~m}^{2} \mathrm{~m}^{-2}$.

In the maize field only few measurements were obtained with LAI-2200, showing a positive bias of more than $50 \%$ in the very low LAI range while for the medium LAI the LoA were closer to 0 as LAI increased. The DHP method only slightly overestimated the direct method (harvest), as shown by a small bias (Fig. 3c, right panel). On the other hand, SS1 underestimated the direct measurements by about $25 \%$ (Fig. 3b, right panel). 


\section{Discussion}

Since direct measurements of LAI are destructive and labour-intensive, indirect methods to assess LAI offer an important alternative. All indirect methods are, however, affected by various factors, as the algorithms and approach used, radiation conditions and zenith angle at the time of measurements, canopy characteristics and clumping (Ryu et al. 2010a). In the current inter-comparison of three indirect methods to measure LAI the different outcome depended on the methods used and the canopy type studied.

\subsection{Explaining the agreement and disagreement among canopy types}

For all four canopy types, the DHP and LAI-2200 methods showed the best agreement, better than each of them compared with SS1. These findings are in line with a previous study (Fang et al. 2014), and may be explained by the similar approach for the clumping correction in both instruments. Differences between DHP and LAI-2200 in the low-to-medium range of LAI (similar to Homolová et al. 2007) may be explained by the gap fraction factor highly sensitive to canopy structure, by leaf distribution and leaf plasticity (Lopez-Lozano et al. 2007). This indicates that clumping at the shoot level might explain the differences between both instruments as documented for a mature Scots pine stand (Jonckheere et al. 2005).

A better agreement between all three indirect methods in the four canopy types was observed in the medium range of LAI. There was a discrepancy between SS1 and the two other indirect methods in both the low (underestimation) and high (overestimation) LAI ranges. The lower values obtained with the SS1 method in the low LAI range, can be explained by the fact that this method did not account for clumping, thus underestimating results (Jones 2014). Clumping factors calculated with the DHP and LAI-2200 methods varied between 0.85 and 0.96 depending on the canopy studied and the method used. These results were in agreement with a previous validation (Ryu et al. 2010a). The lack of a clumping correction in the SS1 method could, thus, only partly account for the observed differences. The higher values obtained with the SS1 method in the medium to high range were possibly related to the levelling-off effect at LAI $5-6 \mathrm{~m}^{2} \mathrm{~m}^{-2}$ in the LAI-2200 and DHP methods, caused by the gap fraction saturation (Gower et al. 1999; Leblanc et al. 2005). Finally, the discrepancy between the SS1 and the other indirect methods might also be due to the fact that the leaf angle distribution was a priori fixed in the SS1 method while it was calculated for each picture (DHP method) or for each set of measurements (LAI-2200 method).

\subsection{Explaining the agreement and disagreement between indirect and direct methods}

In the comparison of the indirect methods with the direct methods, the former overestimated the latter, with exception of SS1 in maize that showed an underestimation in the low-medium range of LAI. There was a clear difference between the two canopies tested, i.e. SRC and maize field. An exponential overestimation was found at the SRC (Fig. 3, right panels), which could be explained: (i) by the WAI in the SRC, and (ii) by the difference in the direct methods used. In the SRC the direct LAI was calculated from litter fall while in the maize field it was calculated from direct harvesting of all plant parts. In addition, the seasonal increment of the WAI in the SRC could explain the increasing difference between the indirect and direct methods. A previous study at the same site showed that the branch area increased with $0.60 \mathrm{~m}^{2} \mathrm{~m}^{-2}$ over three years (Broeckx et al. 2005). The systematic bias (of 25\%) for the medium and high LAI values was in line with a previous report that woody material comprises $5 \%$ to $35 \%$ of the total PAI in forests (Gower et al. 1999).

The slight overestimations of the DHP method compared to the direct measurements in the maize crop agreed very well with earlier findings (Facchi et al. 2010) and might be explained by the presence of the large maize leaves. The underestimation of SS1 in maize was also observed in previous studies (e.g. Wilhelm et al. 2000) and was probably due to the lack of a clumping correction which explained the poor performance of SS1 at low LAI values and in row crops (Chiroro et al. 2006). The chosen ELADP for this canopy type may play an important role, and as the maize leaves change considerably along the growing season, the ELADP value may not have been accurate throughout the entire season (Fang, 2005).

\section{Conclusions}

Although indirect methods to estimate LAI have already been compared in previous studies, large uncertainties still remain. In the current study a standardized approach compared different indirect methods under contrasting canopies, and the results were validated with direct methods using more than one statistical analysis. The use of multiple instruments revealed, on the one hand, a considerable variability and thus, uncertainty in the measurements. On the other hand the approach helped to understand the factors determining LAI, such as the WAI and the degree of clumping, and how these factors differed among methods and canopies. It is, therefore, important to use more than one indirect method for measuring LAI. One suggestion for further research is that an improved hemispherical method is developed to indirectly measure LAI, which is able to simultaneously measure at different heights, providing information about clumping and leaf angle distribution. This improved method should 
furthermore enable to differentiate between non-green and woody parts of the canopy, by infrared techniques, as has been proposed (Jonckheere et al. 2004; Schaefer et al. 2015). For large-scale research infrastructures (as ICOS, LTER, ICP-Forests) a harmonized or standard protocol should enable LAI measurements that can be compared among canopy types, sites and temporal scales.

\section{Acknowledgements}

The research leading to the results of this study has received financial support of the Research Foundation-Flanders (FWO, contract\#GOH3317N), of the University of Tuscia (Italy), of the Methusalem program of the University of Antwerp (Belgium) as well as of the European Commission's Horizon 2020 Research and Innovation Program ENVRIPLUS under grant agreement no. 654182. This study is part of the Ecosystem Thematic Center of the European ICOS infrastructure network. FDM acknowledges support from EU COST Action PROFOUND as a Short Term Scientific Mission under grant agreement FP1304.This study has formed the basis of the Master thesis of FDM under the supervision of DP and MODB. CAC and FDM collected and processed the data, and produced the various drafts of the manuscript contributing equally. All authors contributed in writing and revising the manuscript.

\section{References}

Alivernini, A., Fares, S., Ferrara, C., Chianucci, F., 2018: An objective image analysis method for estimation of canopy attributes from digital cover photography. Trees, 32:713-723.

Bland, J. M., Altman, D. G., 1986: Statistical methods for assessing agreement between two methods of clinical measurement. Lancet, 327:307-310.

Breda, N.J.J., 2003: Ground-based measurements of leaf area index: a review of methods, instruments and current controversies. Journal of Experimental Botany, 54:2403-2417.

Broeckx, L. S., Verlinden, M. S., Ceulemans, R., 2012: Establishment and two-year growth of a bio-energy plantation with fast-growing Populus trees in Flanders (Belgium): effects of genotype and former land use. Biomass \& Bioenergy, 42:151-163.

Broeckx, L. S., Vanbeveren, P. P. S., Verlinden, M. S., Ceulemans, R., 2015: First vs. second rotation of a poplar short rotation coppice: leaf area development, light interception and radiation use efficiency. iForest - Biogeosciences and Forestry, 8:565-573.

Carstensen, B., 2010: Comparing methods of measurement: Extending the LoA by regression. Statistics in Medicine, 29:401-410.

Chen, J. M. R., 1997: Leaf area index of boreal forests: Theory, techniques and measurements. Journal of Geophysical Research, 102:429-443.

Chen, J. M., Black, T. A., 1992: Defining leaf area index for non-flat leaves. Plant, Cell \& Environment, 15:421-429.
Chen, J. M., Rich, P. M., Gower, S. T., Norman, J. M., Plummer, S., 1997: Leaf area index of boreal forests: Theory, techniques, and measurements. Journal of Geophysical Research: Atmospheres, 102:2942929443.

Chianucci, F., Cutini, A., 2013: Estimation of canopy properties in deciduous forests with digital hemispherical and cover photography. Agricultural and Forest Meteorology, 168:130-139.

Chianucci, F., Disperati, L., Guzzi, D., Bianchini, D., Nardino, V., Lastri, C. et al., 2016: Estimation of canopy attributes in beech forests using true colour digital images from a small fixed-wing UAV. International Journal of Applied Earth Observation and Geoinformation, 47:60-68.

Chiroro, D., Milford, J., Makuvaro, V., 2006: An investigation on the utility of the SunScan ceptometer in estimating the leaf area index of a sugarcane canopy. Proceedings of the South African Sugar Technologists Association, 80:143-147.

Cleveland, W. S., 1979: Robust locally weighted regression and smoothing scatterplots. Journal of the American Statistical Association, 74:829-836.

Curiel, Y. J., Konôpka, B., Janssens, I. A., Coenen, K., Xiao, C. W., Ceulemans, R., 2005: Contrasting net primary productivity and carbon distribution between neighbouring stands of Quercus robur and Pinus sylvestris. Tree Physiology, 25:701-712.

Daughtry, C. S. T., 1990: Direct measurements of canopy structure. Remote Sensing Reviews, 5:545-60.

Duchemin, B., Hadriab, R., Errakib, S., Bouleta, G., Maisongrandea, P., Chehbounia, A. et al., 2006: Monitoring wheat phenology and irrigation in Central Morocco: On the use of relationships between evapotranspiration, crops coefficients, leaf area index and remotely-sensed vegetation indices. Agricultural Water Management, 79:1-27.

Facchi, A., Baroni, G., Boschetti, M., Gandolfi, C., 2010: Comparing optical and direct methods for leaf area index determination in a maize crop. Journal of Agricultural Engineering, 1:33-40.

Fang, F., 2005: The retrieval of leaf inclination angle and leaf area index in maize. Master of Science thesis, Geo-Information Science and Earth Observation for Environmental Modelling and Management program. University of Lund, Sweden, $64 \mathrm{p}$.

Fang, H., Li, W., Wei, S., Jiang, C., 2014: Seasonal variation of leaf area index (LAI) over paddy rice fields in NE China: Inter-comparison of destructive sampling, LAI-2200, digital hemispherical photography (DHP), and AccuPAR methods. Agricultural and Forest Meteorology, 198-199:126-14.

Gebauer, R., Cermak, J., Plichta, R., Spinlerova, Z., Urban, J., Volarik, D. et al., 2015: Within-canopy variation in needle morphology and anatomy of vascular tissues in a sparse Scots pine forest. Trees, 29:1447-1457. 
Gielen, B., DeVos, B., Campioli, M., Neirynck, J., Papale, D., Verstraeten, A. et al., 2013: Biometric and eddy covariance-based assessment of decadal carbon sequestration of a temperate Scots pine forest. Agricultural and Forest Meteorology, 174-175:135-143.

Gower, S. T., Kucharik, C. J., Norman, J. M., 1999: Direct and indirect estimation of Leaf Area Index, $\mathrm{f}_{\mathrm{APAR}}$, and Net Primary Production of terrestrial ecosystems. Remote Sensing Environment, 70:29-51.

Homolová, L., Malenovský, Z., Hanuš, J., Tomášková, I., Dvořáková, M., Pokorný, R., 2007: Comparison of different ground techniques to map leaf area index of Norway spruce forest canopy. International Society for Photogrammetry and Remote Sensing (ISPRS), XXXVI, 499-504. [online] URL: http://www.isprs. org/proceedings/XXXVI/7-C50/papers/P95.pdf (accessed 15.01.2019).

Jonckheere, I., Fleck, S., Nackaerts, K., Muys, B., Coppin, P., Weiss, M. et al., 2004: Review of methods for in situ leaf area index determination. Part I: Theories, sensors and hemispherical photography. Agricultural and Forest Meteorology, 121:19-35.

Jonckheere, I., Muys, B., Coppin, P., 2005: Allometry and evaluation of in situ optical LAI determination in Scots pine: a case study in Belgium. Tree Physiology, 25:723-732.

Jones, H. G., 2014: Plants and Microclimate: AQuantitative Approach to Environmental Plant Physiology. Third edition, Cambridge University Press, NY, USA.

Konôpka, B., Pajtík, J., 2014: Similar foliage area but contrasting foliage biomass between young beech and spruce stands. Lesnícky časopis - Forestry Journal, 60:205-213.

Lang, A. R. G., Xiang, Y., 1986: Estimation of leaf area index from transmission of direct sunlight in discontinuous canopies. Agricultural and Forest Meteorology, 37:229-243.

Leblanc, S. G., Chen, J. M., Fernandes, R., Deering, D. W., Conley, A., 2005: Methodology comparison for canopy structure parameters extraction from digital hemispherical photography in boreal forests. Agricultural and Forest Meteorology, 129:187-207.

Lin, A., Zhu, H., Wang, L., Gong, W., Zou, L. 2016: Characteristics of long-term climate change and the ecological responses in central China. Earth Interactions, 20:1-24.

Lopez-Lozano, R., Baret, F., Chelle, M., Rochdi, N., España, M., 2007: Sensitivity of gap fraction to maize architectural characteristics based on 4D model simulations. Agricultural and Forest Meteorology, 143:217-229.

Mason, G. E., Diepstraten, M., Pinjuv, G. L., Lasserre, J-P., 2012: Comparison of direct and indirect leaf area index measurements of Pinus radiate D. Don. Agricultural and Forest Meteorology, 166-167:113-119.
Macfarlane, C., Hoffman, M., Eamus, D., Kerp, N., Higginson, S., McMurtrie, R. et al., 2007: Estimation of leaf area index in eucalypt forest using digital photography. Agricultural and Forest Meteorology, 143:176-188.

Op de Beeck, M., Gielen, B., Jonckheere, I., Samson, R., Janssens, I. A., Ceulemans, R., 2010: Needle agerelated and seasonal photosynthetic capacity variation is negligible for modelling yearly gas exchange of a sparse temperate Scots pine forest. Biogeosciences, 7:199-215.

Passing, H., Bablok, W., 1983: A new biometrical procedure for testing the equality of measurements from two different analytical methods. Application of linear regression procedures for method comparison studies in clinical chemistry, Part I. Journal of Clinical Chemistry and Clinical Biochemistry, 21:709-720.

Ridler, T. W., Calvard, S., 1978: Picture thresholding using an iterative selection method. IEEE Transactions on System, Man and Cybernetics, 8:630-632.

Ryu, Y., Nilson, T., Kobayashi, H., Sonnentag, O., Law, BE., Baldocchi, D. D., 2010a: On the correct estimation of effective leaf area index: Does it reveal information on clumping effects? Agricultural and Forest Meteorology, 150:463-472.

Ryu, Y., Sonnentag, O., Nilson, T., Vargas, R., Kobayashi, H., Wenk, R. et al., 2010b: How to quantify tree leaf area index in an open savanna ecosystem: a multiinstrument and multi-model approach. Agricultural and Forest Meteorology, 150:63-76.

Schaefer, MT., Farmer, E., Soto-Berelov, M., Woodgate, W., Jones, S., 2015: Overview of ground based techniques for estimating LAI. In: Held, A., Phinn, S., Soto-Berelov, M. \& Jones, S. (eds.): AusCover Good Practice Guidelines: A technical handbook supporting calibration and validation activities of remotely sensed data product, 88-118. Version 1.1. TERN AusCover, ISBN 978-0-646-94137-0.

Scrucca, L., 2011: Model-based SIR for dimension reduction. Computational Statistics \& Data Analysis, 55:3010-3026.

Sone, C., Saito, K., Futakuchi, K., 2009: Comparison of three methods for estimating leaf area index of upland rice cultivars. Crop Science, 49:1438-1443.

Thimonier, A., Sedivy, I., Schleppi, P., 2010: Estimating leaf area index in different types of mature forest stands in Switzerland: a comparison of methods. European Journal of Forest Research, 129:543-562.

Verlinden, M. S., Broeckx, L. S., Ceulemans, R., 2015: First vs. second rotation of a poplar short rotation coppice: Above-ground biomass productivity and shoot dynamics. Biomass \& Bioenergy, 73:174-185.

Webb, N., Nichol, C., Wood, J., Potter, E., 2013: User Manual for the SunScan Canopy Analysis System type SS1 Version: 3.0, Delta-T Devices Ltd. 37-39: 49-56. [online] URL: http://www.delta-t.co.uk/ wp-content/uploads/2016/10/SS1-SunScan-UserManual-v2-0.pdf (accessed 15.01.19). 
Weiss, M., Baret, F., Smith, G. J., Jonckheere, I., Coppin, P., 2004: Review of methods for in situ leaf area index (LAI) determination. Part II: Estimation of LAI, errors and sampling. Agricultural and Forest Meteorology, 121:37-53.

Wilhelm, W., Ruwe, K., Schlemmer, M. R., 2000: Comparison of three leaf area index meters in a corn canopy. Crop Science, 40:1179-1183.

Woodgate, W., Jones, S. D., Suarez, L., Hill, M. J., Armston, J. D., Wilkes, P. et al., 2015: Understanding the variability in ground-based methods for retrieving canopy openness, gap fraction, and leaf area index in diverse forest systems. Agricultural and Forest Meteorology, 205:83-95.

Zheng, G. and Moskal, L. M., 2009: Retrieving Leaf Area Index (LAI) using remote sensing: theories, methods and sensors. Sensors, 9:2719-2745.

\section{Other resources:}

GCOS, 2011: Systematic Observation Requirements for Satellite-based Data Products for Climate. WMO, Switzerland. [online] URL:http://www.wmo. int/pages/prog/gcos/Publications/gcos-154.pdf (accessed 15.01.2019).

ICOS Ecosystem Thematic Center. [online] URL: http:// www.icos-etc.eu/icos/ (accessed 15.01.2019).

LI-COR, 2009. LAI-2200 Plant Canopy Analyzer Instruction Manual.Lincoln, NE,USA. [online]URL:https:// www.licor.com/documents/6n3conpja6uj9aq1ruyn (accessed 15.01.2019).

POPFULL Project. [online] URL: http://uahost.uantwerpen.be/popfull/ (accessed 15.01.2019). 


\section{Appendix text and tables}

\section{Appendix text 1. Theory of leaf area index calculation}

In-situ indirect measurements of LAI with optical instruments are based on a statistical and probabilistic approach of the distribution and arrangement of foliar elements (or their complement, the gap fraction) in the canopy. Two theoretical approaches have been developed to infer LAI from optical measurements. With the first approach, LAI is estimated by inversion of the exponential expression of the gap fraction (Eq. 1), i.e. by solving the expression for $L$ (Eq. 2):

$$
\begin{aligned}
& P(\theta)=e^{-G(\theta, \alpha) \Omega(\theta) L / \cos \theta} \\
& L=-(\ln P(\theta) \cos \theta) / G(\theta) \Omega(\theta)
\end{aligned}
$$

where $P(\theta)$ is the gap fraction, $L$ is the LAI, $\theta$ is the zenith angle of view, $\alpha$ is the leaf angle, $G(\theta$, ) corresponds to the fraction of foliage projected on the plane normal to the zenith direction, and $\Omega(\theta)$ is the clumping coefficient, which corrects for a deviation from the assumption of random (Poisson) distribution of canopy elements. When the foliage is randomly distributed within the canopy, $\Omega(\theta)=1$, but as the foliage becomes more clumped, $\Omega(\theta)<1$. The function $G(\theta, \alpha)$ depends on leaf angle distribution, which is generally not known a priori. The calculation of LAI therefore requires gap fraction measurements for a range of zenith angles of view.

The second approach is based on the Beer-Lambert extinction law expanded to plant canopies. This law expresses the attenuation of radiation in a homogenous turbid medium. In such a medium the flux is proportionally absorbed to the optical distance. Assuming a random (Poisson) distribution of leaves within the canopy:

$$
I=I_{0} e^{(-k L)}
$$

where $I_{0}$ is the incident radiation above the canopy, $I$ is the radiation transmitted below the canopy, and $k$ is the extinction coefficient, which depends on the leaf angle distribution and on the direction of the beam (e.g. $k=1$ for entirely horizontal leaves). $I / I_{0}$ is theoretically equivalent to the gap fraction in Eq. 1, i.e. $\mathrm{P}(\theta)$.

Whichever approach used, when applied to canopies with a significant woody-to-total plant area ratio, $L$ represents an overestimation of LAI because of the presence of light-blocking non-leaf and non-green elements. Consequently $L$ provides an estimation of PAI rather than of LAI. To obtain LAI, a woody element correction factor has been introduced (Chen 1997):

$$
L A I=L(1-\alpha)
$$

where $0 \leq \alpha \leq 1$ is the proportion of woody-to-total plant area. Formally $\alpha=$ WAI/PAI where WAI is the wood area index, which can be estimated from destructive sampling by calculating the sum of the hemi-surface area of all branches and stems or taken from the literature, if available.

\section{Appendix text 2. Statistical analyses}

The Passing \& Bablok (1983) regression is based on a robust, non-parametric model and is, unlike the Ordinary Least Squares (OLS) regression, not sensitive towards outliers. It assumes that measurement errors of both methods (direct and indirect) have the same distribution. The 95\% confidence intervals (CI) of the intercept and slope terms of the Passing and Bablok regression equation $y=a+b x$ were interpreted to reveal a constant or a proportional bias. If the $95 \%$ CI for the intercept included the zero value, it could be concluded that there was no significant constant bias. If the $95 \% \mathrm{CI}$ for the slope included 1 as a value, it could be concluded that there was no significant proportional bias. When both conditions were met, one could assume that $y=x$ and that there was no statistically significant difference between the two compared methods. In this latter case the two compared methods could be used interchangeably. In the present study the Passing and Bablok regressions were estimated through the PBreg function of the R MethComp package (Cartensen 2010).

The Bravais-Pearson correlation coefficient (r), which measures the strength of the linear relationship and which was calculated per canopy type, helped the understanding of the previous analysis. This method had to be carefully interpreted as the high $r$ value was not a sufficient condition to affirm that two methods were in agreement; it simply showed the presence of a strong linear relationship between the two methods.

The Bland \& Altman (1986) approach involves a graphical method (hereafter denoted as the B-A plot) consisting of a scatter plot where the difference between each pair of measurements is plotted against the average of each pair of measurements. The mean of the differences is shown on the plot as a reference line indicative of the systematic error (or bias). The $95 \%$ limits of agreement (LoA), which are calculated as the mean of the differences \pm 1.96 standard deviation (SD) of the differences, quantify the range of variability (i.e. precision) between the two measurements. LoA can be evaluated or compared with pre-determined limits to enable the researcher to decide whether given techniques have an acceptable agreement or repeatability. Therefore, assuming a negligible bias, the smaller the LoA, the better the agreement is. In this study an approach based on regressing the relative (\%) differences to the averages was used, and the resulting equation was used to evaluate both the bias and the LoA. The relation between the relative LAI differences and the averages of the differences was estimated through a locally weighted scatterplot smoothing (LOWESS, Cleveland 1979). This is a non-parametric method, which combines much of the simplicity of linear least squares regression with the flexibility of non-linear regression. Fitting is done locally, i.e. at each point $x$ in 
the range of the data set a low-degree polynomial is fitted to a subset of the data using points in a neighbourhood of $x$, weighted by their distance from $x$. The LOWESS

Table A1. Abbreviations and symbols - in the main text

$\begin{array}{ll}\text { Abbreviation } & \text { Definition } \\ \text { ACF } & \text { Apparent Clumping Factor } \\ \text { B-A } & \text { Bland and Altman } \\ \text { CI } & \text { Confidence Interval } \\ \text { DHP } & \text { Digital Hemispherical Photography } \\ \text { ELADP } & \text { Ellipsoidal Leaf Angle Parameter } \\ \text { LA } & \text { Total Leaf Area } \\ \text { LAI } & \text { Leaf Area Index } \\ \text { LAI-2200 } & \text { LAI-2200 Plant Canopy Analyzer } \\ \text { LAI } & \text { Maximum LAI } \\ \text { LoA } & \text { Limits of Agreement } \\ \text { PAI } & \text { Plant Area Index } \\ \text { PAR } & \text { Photosynthetically Active Radiation } \\ \text { P-B } & \text { Passing \& Bablok } \\ \text { OLS } & \text { Ordinary Least Squares regression } \\ \text { SLA } & \text { Specific Leaf Area } \\ \text { SRC } & \text { Short Rotation Coppice } \\ \text { SS1 } & \text { SunScan Canopy Analysis System } \\ \text { TLS } & \text { Terrestrial Laser Scanning } \\ \text { UAV } & \text { Unmanned Aerial Vehicle }\end{array}$

curve was computed through the loees.s $d$ function implemented in the msir $R$ package (Scrucca 2011).

Table A2. Abbreviations and symbols - in the Appendix text

$\begin{array}{lll}\text { Abbreviation } & \text { Definition } & \text { Additional information } \\ \mathrm{C} & \text { Constant } & - \\ \mathrm{G}(\theta, \alpha) & \text { Fraction of foliage projected on the plane normal to the zenith direction } & - \\ \mathrm{I} & \text { Radiation transmitted } & - \\ \mathrm{r} & \text { Bravais-Pearson correlation coefficient } & - \\ \mathrm{I} / \mathrm{I}_{0} & \text { Theoretically equivalent to the gap fraction } & - \\ \mathrm{K} & \text { Extinction coefficient } & \text { Represents LAI in the equation } \\ \mathrm{L} & \text { The LAI } & - \\ \mathrm{P} & \text { Proportional } & - \\ \mathrm{P}(\theta) & \text { The gap fraction } & - \\ \mathrm{SD} & \text { Standard Deviation } & - \\ \Theta & \text { The zenith angle of view } & - \\ \Omega(\theta) & \text { Clumping coefficient } & \end{array}$

